\title{
Interval Estimation For The Scale Parameter Of Burr Type X Distribution Based On Grouped Data
}

\author{
Amjad D. Al-Nasser \\ Yarmouk University, Jordan, amjadn@yu.edu.jo \\ Ayman Baklizi \\ Yarmouk University,Irbid, Jordan, baklizi1@hotmail.com
}

Follow this and additional works at: http:// digitalcommons.wayne.edu/jmasm

Part of the Applied Statistics Commons, Social and Behavioral Sciences Commons, and the Statistical Theory Commons

\section{Recommended Citation}

Al-Nasser, Amjad D. and Baklizi, Ayman (2004) "Interval Estimation For The Scale Parameter Of Burr Type X Distribution Based On Grouped Data," Journal of Modern Applied Statistical Methods: Vol. 3 : Iss. 2 , Article 11.

DOI: $10.22237 /$ jmasm/1099267860

Available at: http://digitalcommons.wayne.edu/jmasm/vol3/iss2/11 


\title{
Interval Estimation For The Scale Parameter Of Burr Type X Distribution Based On Grouped Data
}

\author{
Amjad Al-Nasser Ayman Baklizi \\ Department of Statistics \\ Yarmouk University, Jordan
}

The application of some bootstrap type intervals for the scale parameter of the Burr type X distribution with grouped data is proposed. The general asymptotic confidence interval procedure (Chen \& Mi, 2001) is studied. The performance of these intervals is investigated and compared. Some of the bootstrap intervals give better performance for situations of small sample size and heavy censoring.

Key words: Bootstrap, Burr type X distribution, grouped data, interval estimation

\section{Introduction}

In many applications, individual observations are very naturally categorized into mutually exclusive and exhaustive groups; such type of data is often called grouped data. Grouped data arise frequently in life testing experiments when inspecting the test units intermittently for failure, this procedure is frequently used because it requires less testing effort than continuous inspection. The data obtained from intermittent inspection consists only of the number of failures in each inspection interval. Other examples of natural occurrences of grouped data are given in Pettitt and Stephens (1977). In this article, a different computer intensive confidence interval is obtained based on grouped data for the scale parameter of Burr Type $X$ Distribution “ $\operatorname{Burr} X(v, \theta)$ ” whose distribution function is given by

Amjad D. Al-Nasser, Department of Statistics, Faculty of Science. Yarmouk University, Irbid, Jordan. Email: amjadn@yu.edu.jo. Ayman Baklizi is Assistant Professor of Applied Statistics. His research interests are in accelerated life tests and censored data. Email: baklizi1@hotmail.com.

$$
F(x, v, \theta)=\left[1-e^{-\left(\frac{x}{\theta}\right)^{2}}\right]^{v}, \quad x>0, \theta>0, \quad v>0
$$

By taking the first derivative on (1), the density function of the BurrX distribution can be obtained as:

$$
f(x, v, \theta)=\frac{2 v x}{\theta^{2}}\left(e^{-\left(\frac{x}{\theta}\right)^{2}}\right)\left(1-e^{-\left(\frac{x}{\theta}\right)^{2}}\right)^{v-1}
$$

This density function introduced by Burr (1942) providing new model of life time data. The applications of the Burr distribution may be found in the literature for the different twelve types of this distribution. Various authors, considered BurrX in different aspects (e. g., Mudholkar et al., 1995; Mudholkar \& Hutson, 1996; Jaheen, 1995, 1996; Surles \&Padgett, 1998, 2001; Ahmad et al., 1997). This distribution is a generalized Rayleigh distribution and also it is considered as a special case of exponentiated Weibull distribution that introduced by Mudholkar and Sirvastava (1993). The shape of this distribution depends on the parameter $v$, by increasing its value the more symmetry of the distribution. Figure 1 represents the BurrX density function with $v=0.4,0.8,1.2$, 1.6 and 2 with unity scale: 
Figure 1 . Burr $X$ density with unity scale and different shaper parameters values.

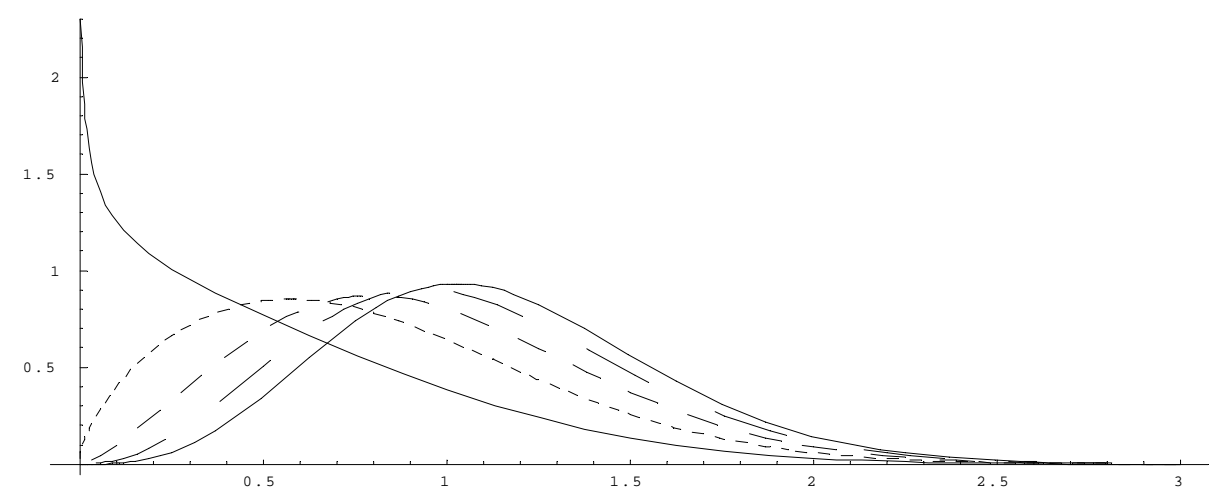

In this article, bootstrap methods are used to construct confidence intervals for the scale parameter of BurrX distribution, $\theta$. Many authors consider bootstrap methods in a vast range of domains (e. g., Davison et al., 2003; Noreen, 1989; Hall, 1988, 1992; Mooney \& Duval, 1989). However, the widely used methods in constructing the confidence intervals consist of the second order accurate bootstrap confidence intervals, namely; "Bootstrap-t (BST)" which give good theoretical coverage probabilities but not reliable and the "Bias Corrected and Accelerated (BCa)" which is the second improved version of the Percentile intervals (PRC). These methods may provide good approximate confidence intervals and better than the usual standard intervals (Efron, 2003). We applied these methods, in addition to, the first improved version of PRC which is the Bias-corrected (BC). Other methods are used, Jackknife Intervals (JAC) and Intervals Based on the Bootstrap Standard Deviation (BSD) Intervals.

We review the approximated confidence interval proposed by Chen and Mi (2001). Next, different bootstrap confidence interval approaches are considered. Then, Monte Carlo evidence on the numerical performance of the bootstrap is presented. Finally, make recommendations.
Approximated Confidence Interval

This confidence interval was proposed by Chen and Mi (2001). They introduced an approximate confidence intervals of certain parameters for distribution function on $(0, \infty)$ which satisfy some monotonic conditions using grouped data. To explain their method, let $X_{1}, X_{2}, \ldots, X_{n}$ be a random sample from a distribution $F(X, \theta)$ of a continuous type having probability density on $(0, \infty)$, where $\theta$ is unknown parameter. Assume that there are $\mathrm{K}$ (groups) inspection times $0<t_{1}<t_{2}<\ldots<t_{k}<\infty$, and the probability of an observation fail in the ith group is $p_{i}=P\left(t_{i-1}<X \leq t_{i}\right), i=1,2, \ldots, K+1 \quad$ where $t_{0}=0$ and $t_{k+1}=\infty$. Let $r_{i}, i=1,2, \ldots, K+1$ is the number of observations fail in the $\mathrm{i}^{\text {th }}$ interval $\left[t_{i-1}, t_{i}\right)$. Based on the upper endpoint approach they define the random variable

$$
\zeta_{n}=\sum_{i=1}^{k} r_{i} t_{i}+r_{k+1} t_{k}
$$

it follows that from the Central Limit Theorem

$$
\frac{\zeta_{n}-n g(\vartheta)}{\sqrt{n} \sigma(\theta)} \rightarrow N(0,1) \text { as } n \rightarrow \infty
$$


where $g(\theta)=\sum_{i=1}^{k} t_{i} p_{i}+t_{k} p_{k+1}$,

and

$\sigma^{2}(\theta)=\left(\sum_{i=1}^{k} t_{i}^{2} p_{i}+t_{k}^{2} p_{k+1}\right)-\left(\sum_{i=1}^{k} t_{i} p_{i}+t_{k} p_{k+1}\right)^{2}$.

An approximate confidence interval for $\theta$ can be obtained based on the following estimates;

$$
\hat{p}_{i}=\frac{r_{i}}{n}, i=1,2, \ldots, K+1
$$

and

$s_{n}^{2}=\left(\sum_{i=1}^{k} t_{i}^{2} \hat{p}_{i}+t_{k}^{2} \hat{p}_{k+1}\right)-\left(\sum_{i=1}^{k} t_{i} \hat{p}_{i}+t_{k} \hat{p}_{k+1}\right)^{2}$

for $p_{i}$ and $\sigma^{2}(\theta)$, respectively. It follows that, asymptotically:

$$
p\left(\frac{\zeta_{n}}{n}-Z_{\alpha / 2} \frac{s_{n}}{\sqrt{n}}<g(\vartheta)<\frac{\zeta_{n}}{n}+Z_{\alpha / 2} \frac{s_{n}}{\sqrt{n}}\right)=1-\alpha .
$$

When the function $g(\theta)$ is monotone, an approximate $(1-\alpha) \%$ confidence interval for $\theta$, call it the CM interval, can be obtained as:

$$
\left[g^{-1}\left(\frac{\zeta_{n}}{n}-z_{\alpha / 2} \frac{S_{n}}{\sqrt{n}}\right), g^{-1}\left(\frac{\zeta_{n}}{n}+z_{\alpha / 2} \frac{s_{n}}{\sqrt{n}}\right)\right]
$$

However, the above interval possesses exact coverage probabilities and symmetry probabilities only for sufficiently large sample sizes.

\section{Methodology}

The bootstrap is a nonparametric technique introduced by Efron in 1979. In this study, we consider six bootstrap methods to construct confidence intervals for the scale parameter of BurrX distribution, $\theta$. For each one of the methods described below, the random variable $\zeta_{n}$ as defined in (2) calculated from the original data. We generate $B$ bootstrap series $x^{* 1}, x^{* 2}, \cdots, x^{* B}$ and then $\zeta_{n}^{*}$ be calculated from the bootstrap sample for each series. The standard normal cumulative distribution function is denoted by $\Phi($.$) , and z_{\alpha}$ is the $\alpha$ percentile of the standard normal distribution.

t Interval (BTS Intervals)

BST is very similar to confidence intervals based on the t-Student distribution . To construct this interval let $z_{\alpha}^{*}$ be the $\alpha$ percentile of the empirical distribution of $Z^{*}=\frac{\left(\zeta_{n}^{*}-\zeta_{n}\right)}{s_{n}^{*}}$, where $s_{n}^{*}$ is estimated standard error of $\zeta_{n}^{*}$ calculated from the bootstrap sample. Then BST interval for $\theta$ is given by

$$
\left\lfloor g^{-1}\left(\zeta_{n}-z_{\alpha / 2}^{*} S_{n}^{*}\right), g^{-1}\left(\zeta_{n}+z_{1-\alpha / 2}^{*} S_{n}^{*}\right)\right\rfloor
$$

The Percentile Interval (PRC Interval)

An alternative bootstrap interval has been proposed in Efron (1979), and is discussed in detail in Efron and Tibshirani (1993). Let $\hat{g}$ be the cumulative distribution function of $\zeta_{n}^{*}$, then the $1-\alpha$ PRC interval is given by

$$
\left[\hat{g}^{-1}\left(\frac{\alpha}{2}\right), \hat{g}^{-1}\left(1-\frac{\alpha}{2}\right)\right]
$$

The Bias Corrected Interval (Interval)

The bias corrected interval (Efron, 1982) is calculated using the corrected percentiles of the bootstrap distribution of $\zeta_{n}^{*}$. The determination of the appropriate percentiles depends on a number $\left(\hat{z}_{0}=\Phi^{-1}\left(\frac{\#\left\{\zeta_{n}^{*}<\zeta_{n}\right\}}{B}\right)\right.$ ) which measure the median bias and called the bias correction. The $1-\alpha \mathrm{BC}$ interval is given by

$$
\left[\hat{g}^{-1}\left(\alpha_{1}\right), \hat{g}^{-1}\left(\alpha_{2}\right)\right]
$$


where

$$
\begin{gathered}
\alpha_{1}=\Phi\left(2 \hat{z}_{0}+z_{\alpha / 2}\right), \\
\alpha_{2}=\Phi\left(2 \hat{z}_{0}+z_{1-\alpha / 2}\right) .
\end{gathered}
$$

Bootstrap Standard Deviation (BSD Intervals)

An interval similar in form to the based on the jackknife can be constructed as follows;

$$
\left\lfloor\zeta_{n}-z_{\alpha / 2} s \tilde{e}, \zeta_{n}+z_{1-\alpha / 2} s \tilde{e}\right\rfloor
$$

Bias Corrected and Accelerated Interval (BCa Interval)

In this method, we calculate the bias correction $\hat{z}_{0}$ as before. We need to calculate the acceleration:

$$
\hat{a}=\frac{\sum_{i=1}^{n}\left(\zeta_{n}(.)-\zeta_{n}(i)\right)^{3}}{6\left\{\sum_{i=1}^{n}\left(\zeta_{n}(.)-\zeta_{n}(i)\right)^{2}\right\}^{3 / 2}} .
$$

$$
\begin{gathered}
s \tilde{e}^{2}=\frac{1}{B-1} \sum_{i=1}^{B}\left(\zeta_{i, n}^{*}-\bar{\zeta}_{n}^{*}\right)^{2}, \\
\bar{\zeta}_{n}^{*}=\frac{1}{B} \sum_{i=1}^{B} \zeta_{i, n}^{*}
\end{gathered}
$$

is the bootstrap estimate of the variance of $\zeta_{n}$.

\section{Results}

Thus in the same way we calculated the $\mathrm{BC}$ interval; the $1-\alpha \mathrm{BCa}$ interval is given by

$$
\left[\hat{g}^{-1}\left(\alpha_{1}\right), \hat{g}^{-1}\left(\alpha_{2}\right)\right]
$$

where

$$
\begin{gathered}
\alpha_{1}=\Phi\left(\hat{z}_{0}+\frac{\hat{z}_{0}+z_{\alpha / 2}}{1-\hat{a}\left(\hat{z}_{0}+z_{\alpha / 2}\right)}\right), \\
\alpha_{2}=\Phi\left(\hat{z}_{0}+\frac{\hat{z}_{0}+z_{1-\alpha / 2}}{1-\hat{a}\left(\hat{z}_{0}+z_{1-\alpha / 2}\right)}\right) ;
\end{gathered}
$$

and $\zeta_{n}(i)$ is calculated using the original data excluding the i-th observation and

$$
\zeta_{n}(.)=\frac{\sum_{i=1}^{n} \zeta_{n}(i)}{n} \text {. }
$$

Jackknife Intervals (JAC Intervals)

An interval based on the jackknife (Efron \&Tibshirani, 1993) can be constructed as follows;

$$
\left\lfloor\zeta_{n}(.)-z_{\alpha / 2} s \hat{e}, \zeta_{n}(.)+z_{1-\alpha / 2} s \hat{e}\right\rfloor
$$

where

$$
s \hat{e}^{2}=\frac{n-1}{n} \sum_{i=1}^{n}\left(\zeta_{n}(.)-\zeta_{n}(i)\right)^{2}
$$

is the jackknife estimate of the variance of $\zeta_{n}$.

A simulation study is conducted to investigate the performance of the interval methods. The 95\% confidence intervals for $\theta$ was constructed using the seven methods proposed in (3)- (9). The criterion of attainment of lower and upper error probabilities (Jennings, 1987) which are both taken equal to 0.025 was used. In order to compare the performance of the bootstrap estimates, 2000 samples were generated from the BurrX distribution with $\theta=1$ and $v=0.4$, $0.8,1.2,1.6$ and 2; with different sizes $\mathrm{n}=20$, 30, 50 and 100 .

For each combination, each sample was divided into $(K+1)$ groups where $K=2$, 4 and 8 . The censoring proportion (cp) is taken as 0.2 , 0.4 and 0.6. The empirical bootstrap distribution was constructed using $\mathrm{B}=2000$ replications. Then, the following quantities are simulated for each interval: Lower error rates $(\mathrm{L})$ : The fraction of intervals that fall entirely above the true parameter; Upper error rates (U): The fraction of intervals that fall entirely below the true parameter and Total error rates (T): The fraction of intervals that did not contain the true parameter value. The results are given in Tables 1-3.

\section{Conclusion}

We have compared the performance of several versions of bootstrap confidence intervals together and with the approximated (CM) confidence interval. Bootstrap confidence intervals outperform the CM interval in terms of 
total error rates and symmetry in many cases with large sample sizes and appear to be better for small sample sizes.

It can be noted that for $\mathrm{k}=2$, small sample size $(\mathrm{n}=20,30)$ and with censoring proportion ( $\mathrm{cp}=0.4)$, the CM intervals tend to be anti-conservative. This is also true for JAC, BTS and BCa intervals. On the other hand, the $\mathrm{BC}, \mathrm{BSD}$ and PRC intervals tend to attain the nominal sizes. As the censoring proportion is light to moderate with $\mathrm{cp}=0.8$, the JAC and the $\mathrm{CM}$ intervals tend to be equivalent and grossly anti-conservative while the $\mathrm{BC}$ and $\mathrm{BCa}$ intervals tend to be grossly conservative. For larger sample sizes $(n=50,100)$ all intervals attain their nominal sizes except for the BC and $\mathrm{BCa}$ intervals where they remain anticonservative. In situations where $\mathrm{k}=2$ and small sample size, all intervals are asymmetric. As $\mathrm{k}$ increases, the intervals tend generally to be more symmetric.

The performance of the PRC, BC and BCa intervals improves considerably for larger values of $\mathrm{k}$. Also their performance improves for higher values of $r$, that is, the more symmetric the parent BurrX distribution, the more symmetric the PRC, BC and BCa intervals tend to be. In conclusion, it appears that the intervals proposed by Chen and Mi (2001) have a good performance except for situations of small sample size and heavy censoring. In this case the BTS, JAC and especially BSD intervals provide better alternatives.

\section{References}

Chen, Z., \& Mi, J. (2001). An Approximate confidence interval for the scale parameter of the gamma distribution based on grouped data. Statistical Papers, 42, 285-299.
Davison., A. C., Hinkley, D. V., \& Young. G. A. (2003). Recent developments in bootstrap methodology. Statistical Science, 18(2), 141-157.

Efron B. (1979). Bootstrap methods: another look at the jackknife. The Annals of Statistics, 7, 1-26.

Efron, B. (1982). The jackknife, the bootstrap and other resampling plans. Philadelphia: SIAM.

Efron, B., \& Tibshirani, R. (1993). An introduction to the bootstrap. New York: Chapman and Hall.

Efron B. (2003). Second thoughts in the Bootstrap. Statistical Science, 18(2), 135-140.

Hall, P. (1992). The bootstrap and

Edgeworth expansion. NY: Springer-Verlag.

Hall, P. (1988). Theoretical comparison of bootstrap confidence intervals. Annals of Statistics, 16, 927-953.

Jennings, D. (1987). How do we judge confidence intervals adequacy? The American Statistician, 41(4), 335-337.

Kulldorff, G. (1961). Estimation from grouped and partially grouped samples. New York: Wiley.

Mudholker, G. S., \& Srivastava, D. K. (1993). Exponentiated Weibull family for analyzing bathtub failure-rate data. IEEE Transactions on Reliability, 42. 299-302.

Mooney, C. \& Duval, R. (1993). Bootstrapping a nonparametric approach to statistical inference. London: Sage Publications.

Noreen, E. W. (1989). Computer intensive methods for testing hypotheses. New York: John Wiley and Sons.

Surles, J. G., \& Padgett, W. J. (2001). Inference for reliability and stress-strength for a scaled Burr Type X Distribution. Lifetime Data Analysis, 7, 187-200. 
Table.1 95\% confidence interval for $\theta$ based on $\mathrm{k}=2$.

\begin{tabular}{|c|c|c|c|c|c|c|c|c|c|c|c|c|c|c|}
\hline & & $\mathrm{n}$ & \multicolumn{3}{|c|}{20} & \multicolumn{3}{|c|}{30} & \multicolumn{3}{|c|}{50} & \multicolumn{3}{|c|}{100} \\
\hline $\mathrm{R}$ & CP & Method & $\mathrm{L}$ & $\mathrm{U}$ & $\mathrm{T}$ & $\mathrm{L}$ & $\mathrm{U}$ & $\mathrm{T}$ & $\mathrm{L}$ & $\mathrm{U}$ & $\mathrm{T}$ & $\mathrm{L}$ & $\mathrm{U}$ & $\mathrm{T}$ \\
\hline \multirow[t]{21}{*}{0.4} & \multirow[t]{7}{*}{0.4} & $\mathrm{CM}$ & 0.000 & 0.023 & 0.023 & 0.020 & 0.019 & 0.039 & 0.012 & 0.027 & 0.039 & 0.015 & 0.027 & 0.042 \\
\hline & & BTS & 0.000 & 0.008 & 0.008 & 0.020 & 0.011 & 0.031 & 0.012 & 0.010 & 0.022 & 0.015 & 0.040 & 0.055 \\
\hline & & PRC & 0.000 & 0.026 & 0.026 & 0.000 & 0.045 & 0.045 & 0.000 & 0.096 & 0.096 & 0.002 & 0.087 & 0.088 \\
\hline & & BC & 0.000 & 0.070 & 0.070 & 0.000 & 0.048 & 0.048 & 0.012 & 0.066 & 0.078 & 0.052 & 0.035 & 0.087 \\
\hline & & BCA & 0.000 & 0.085 & 0.085 & 0.000 & 0.068 & 0.068 & 0.008 & 0.089 & 0.097 & 0.040 & 0.047 & 0.087 \\
\hline & & JAC & 0.000 & 0.023 & 0.023 & 0.020 & 0.019 & 0.039 & 0.012 & 0.027 & 0.039 & 0.042 & 0.027 & 0.069 \\
\hline & & BSD & 0.006 & 0.046 & 0.052 & 0.013 & 0.024 & 0.037 & 0.025 & 0.025 & 0.050 & 0.020 & 0.030 & 0.050 \\
\hline & \multirow[t]{7}{*}{0.6} & $\mathrm{CM}$ & 0.013 & 0.024 & 0.036 & 0.007 & 0.026 & 0.033 & 0.017 & 0.029 & 0.045 & 0.023 & 0.022 & 0.045 \\
\hline & & BTS & 0.013 & 0.009 & 0.022 & 0.007 & 0.020 & 0.027 & 0.017 & 0.005 & 0.022 & 0.022 & 0.020 & 0.041 \\
\hline & & PRC & 0.000 & 0.009 & 0.009 & 0.002 & 0.023 & 0.025 & 0.005 & 0.027 & 0.031 & 0.006 & 0.037 & 0.043 \\
\hline & & BC & 0.013 & 0.082 & 0.095 & 0.008 & 0.059 & 0.067 & 0.041 & 0.043 & 0.083 & 0.029 & 0.036 & 0.064 \\
\hline & & BCA & 0.000 & 0.083 & 0.083 & 0.007 & 0.064 & 0.071 & 0.041 & 0.046 & 0.087 & 0.023 & 0.042 & 0.065 \\
\hline & & JAC & 0.013 & 0.024 & 0.036 & 0.007 & 0.026 & 0.033 & 0.017 & 0.029 & 0.045 & 0.023 & 0.022 & 0.045 \\
\hline & & BSD & 0.018 & 0.037 & 0.054 & 0.016 & 0.033 & 0.049 & 0.022 & 0.021 & 0.043 & 0.021 & 0.027 & 0.048 \\
\hline & \multirow[t]{7}{*}{0.8} & $\mathrm{CM}$ & 0.029 & 0.049 & 0.078 & 0.018 & 0.024 & 0.042 & 0.015 & 0.027 & 0.042 & 0.024 & 0.034 & 0.058 \\
\hline & & BTS & 0.028 & 0.017 & 0.044 & 0.018 & 0.007 & 0.025 & 0.017 & 0.011 & 0.027 & 0.024 & 0.016 & 0.039 \\
\hline & & PRC & 0.006 & 0.017 & 0.022 & 0.005 & 0.023 & 0.028 & 0.006 & 0.015 & 0.021 & 0.019 & 0.016 & 0.034 \\
\hline & & BC & 0.006 & 0.091 & 0.097 & 0.008 & 0.101 & 0.109 & 0.013 & 0.091 & 0.104 & 0.037 & 0.056 & 0.093 \\
\hline & & BCA & 0.003 & 0.091 & 0.094 & 0.008 & 0.104 & 0.112 & 0.008 & 0.093 & 0.101 & 0.031 & 0.057 & 0.088 \\
\hline & & JAC & 0.029 & 0.049 & 0.078 & 0.018 & 0.024 & 0.042 & 0.015 & 0.027 & 0.042 & 0.024 & 0.034 & 0.058 \\
\hline & & BSD & 0.022 & 0.043 & 0.064 & 0.018 & 0.043 & 0.061 & 0.017 & 0.029 & 0.046 & 0.024 & 0.033 & 0.057 \\
\hline \multirow[t]{14}{*}{0.8} & \multirow[t]{7}{*}{0.4} & $\mathrm{CM}$ & 0.000 & 0.020 & 0.020 & 0.024 & 0.021 & 0.045 & 0.014 & 0.027 & 0.041 & 0.019 & 0.023 & 0.042 \\
\hline & & BTS & 0.000 & 0.005 & 0.005 & 0.024 & 0.015 & 0.039 & 0.014 & 0.009 & 0.023 & 0.019 & 0.038 & 0.057 \\
\hline & & PRC & 0.000 & 0.022 & 0.022 & 0.000 & 0.050 & 0.050 & 0.000 & 0.090 & 0.090 & 0.001 & 0.086 & 0.087 \\
\hline & & $\mathrm{BC}$ & 0.000 & 0.060 & 0.060 & 0.001 & 0.052 & 0.053 & 0.014 & 0.068 & 0.082 & 0.059 & 0.032 & 0.091 \\
\hline & & BCA & 0.000 & 0.078 & 0.078 & 0.000 & 0.076 & 0.076 & 0.013 & 0.086 & 0.098 & 0.048 & 0.044 & 0.092 \\
\hline & & JAC & 0.000 & 0.020 & 0.020 & 0.024 & 0.021 & 0.045 & 0.014 & 0.027 & 0.041 & 0.041 & 0.023 & 0.064 \\
\hline & & BSD & 0.007 & 0.037 & 0.043 & 0.016 & 0.033 & 0.049 & 0.021 & 0.028 & 0.049 & 0.026 & 0.031 & 0.056 \\
\hline & \multirow[t]{7}{*}{0.6} & $\mathrm{CM}$ & 0.012 & 0.027 & 0.039 & 0.014 & 0.023 & 0.037 & 0.018 & 0.040 & 0.058 & 0.022 & 0.028 & 0.050 \\
\hline & & BTS & 0.012 & 0.011 & 0.022 & 0.014 & 0.015 & 0.029 & 0.018 & 0.011 & 0.029 & 0.022 & 0.025 & 0.046 \\
\hline & & PRC & 0.000 & 0.011 & 0.011 & 0.001 & 0.019 & 0.020 & 0.008 & 0.036 & 0.044 & 0.007 & 0.044 & 0.051 \\
\hline & & BC & 0.012 & 0.076 & 0.088 & 0.014 & 0.046 & 0.059 & 0.047 & 0.057 & 0.104 & 0.027 & 0.042 & 0.068 \\
\hline & & BCA & 0.000 & 0.077 & 0.077 & 0.014 & 0.050 & 0.063 & 0.047 & 0.062 & 0.108 & 0.022 & 0.050 & 0.072 \\
\hline & & JAC & 0.012 & 0.027 & 0.039 & 0.014 & 0.023 & 0.037 & 0.018 & 0.040 & 0.058 & 0.022 & 0.028 & 0.050 \\
\hline & & BSD & 0.017 & 0.035 & 0.052 & 0.023 & 0.037 & 0.060 & 0.024 & 0.041 & 0.065 & 0.025 & 0.035 & 0.060 \\
\hline & \multirow[t]{7}{*}{0.8} & CM & 0.029 & 0.072 & 0.100 & 0.018 & 0.032 & 0.050 & 0.020 & 0.035 & 0.055 & 0.023 & 0.030 & 0.053 \\
\hline & & BTS & 0.028 & 0.022 & 0.050 & 0.018 & 0.013 & 0.030 & 0.023 & 0.010 & 0.032 & 0.023 & 0.019 & 0.042 \\
\hline & & PRC & 0.007 & 0.022 & 0.029 & 0.003 & 0.031 & 0.034 & 0.011 & 0.018 & 0.028 & 0.015 & 0.019 & 0.033 \\
\hline & & BC & 0.008 & 0.107 & 0.115 & 0.005 & 0.097 & 0.102 & 0.018 & 0.099 & 0.117 & 0.035 & 0.050 & 0.085 \\
\hline & & BCA & 0.005 & 0.108 & 0.112 & 0.005 & 0.099 & 0.104 & 0.012 & 0.100 & 0.112 & 0.028 & 0.051 & 0.078 \\
\hline & & JAC & 0.029 & 0.072 & 0.100 & 0.018 & 0.032 & 0.050 & 0.020 & 0.035 & 0.055 & 0.023 & 0.030 & 0.053 \\
\hline & & BSD & 0.025 & 0.054 & 0.079 & 0.020 & 0.049 & 0.069 & 0.026 & 0.041 & 0.066 & 0.023 & 0.030 & 0.053 \\
\hline
\end{tabular}


Table 1 Continued:

\begin{tabular}{|c|c|c|c|c|c|c|c|c|c|c|c|c|c|c|}
\hline \multirow[t]{7}{*}{1.2} & \multirow[t]{7}{*}{0.4} & $\mathrm{CM}$ & 0.000 & 0.022 & 0.022 & 0.026 & 0.019 & 0.045 & 0.014 & 0.030 & 0.043 & 0.022 & 0.022 & 0.043 \\
\hline & & BTS & 0.000 & 0.006 & 0.006 & 0.026 & 0.013 & 0.039 & 0.014 & 0.011 & 0.025 & 0.022 & 0.036 & 0.058 \\
\hline & & PRC & 0.000 & 0.026 & 0.026 & 0.000 & 0.047 & 0.047 & 0.000 & 0.088 & 0.088 & 0.000 & 0.076 & 0.076 \\
\hline & & BC & 0.000 & 0.066 & 0.066 & 0.016 & 0.054 & 0.069 & 0.014 & 0.062 & 0.076 & 0.042 & 0.036 & 0.078 \\
\hline & & BCA & 0.000 & 0.079 & 0.079 & 0.001 & 0.077 & 0.078 & 0.014 & 0.077 & 0.091 & 0.033 & 0.042 & 0.074 \\
\hline & & JAC & 0.000 & 0.022 & 0.022 & 0.026 & 0.019 & 0.045 & 0.014 & 0.030 & 0.043 & 0.044 & 0.022 & 0.066 \\
\hline & & BSD & 0.011 & 0.040 & 0.051 & 0.018 & 0.026 & 0.044 & 0.019 & 0.026 & 0.045 & 0.024 & 0.023 & 0.047 \\
\hline & \multirow[t]{7}{*}{0.6} & $\mathrm{CM}$ & 0.013 & 0.029 & 0.042 & 0.008 & 0.017 & 0.025 & 0.015 & 0.034 & 0.048 & 0.022 & 0.022 & 0.044 \\
\hline & & BTS & 0.013 & 0.014 & 0.026 & 0.008 & 0.015 & 0.023 & 0.015 & 0.008 & 0.023 & 0.022 & 0.016 & 0.038 \\
\hline & & PRC & 0.000 & 0.014 & 0.014 & 0.001 & 0.016 & 0.017 & 0.004 & 0.030 & 0.034 & 0.005 & 0.041 & 0.046 \\
\hline & & $\mathrm{BC}$ & 0.013 & 0.083 & 0.095 & 0.008 & 0.041 & 0.049 & 0.038 & 0.036 & 0.073 & 0.029 & 0.043 & 0.072 \\
\hline & & BCA & 0.006 & 0.083 & 0.088 & 0.008 & 0.041 & 0.049 & 0.035 & 0.045 & 0.079 & 0.026 & 0.049 & 0.074 \\
\hline & & JAC & 0.013 & 0.029 & 0.042 & 0.008 & 0.017 & 0.025 & 0.015 & 0.034 & 0.048 & 0.022 & 0.022 & 0.044 \\
\hline & & BSD & 0.016 & 0.038 & 0.054 & 0.021 & 0.029 & 0.050 & 0.020 & 0.030 & 0.050 & 0.021 & 0.029 & 0.050 \\
\hline & \multirow[t]{7}{*}{0.8} & CM & 0.020 & 0.062 & 0.081 & 0.012 & 0.032 & 0.043 & 0.018 & 0.027 & 0.045 & 0.020 & 0.037 & 0.056 \\
\hline & & BTS & 0.019 & 0.019 & 0.037 & 0.012 & 0.013 & 0.024 & 0.019 & 0.010 & 0.028 & 0.020 & 0.019 & 0.038 \\
\hline & & PRC & 0.004 & 0.019 & 0.022 & 0.003 & 0.032 & 0.035 & 0.007 & 0.017 & 0.023 & 0.014 & 0.019 & 0.032 \\
\hline & & BC & 0.004 & 0.139 & 0.143 & 0.004 & 0.145 & 0.148 & 0.018 & 0.095 & 0.113 & 0.022 & 0.054 & 0.076 \\
\hline & & BCA & 0.004 & 0.139 & 0.143 & 0.003 & 0.146 & 0.149 & 0.011 & 0.096 & 0.106 & 0.016 & 0.055 & 0.071 \\
\hline & & JAC & 0.020 & 0.062 & 0.081 & 0.012 & 0.032 & 0.043 & 0.018 & 0.027 & 0.045 & 0.020 & 0.037 & 0.056 \\
\hline & & BSD & 0.017 & 0.055 & 0.071 & 0.014 & 0.049 & 0.063 & 0.022 & 0.031 & 0.053 & 0.018 & 0.029 & 0.046 \\
\hline \multirow[t]{14}{*}{1.6} & \multirow[t]{7}{*}{0.4} & $\mathrm{CM}$ & 0.000 & 0.024 & 0.024 & 0.029 & 0.016 & 0.045 & 0.014 & 0.033 & 0.046 & 0.017 & 0.020 & 0.036 \\
\hline & & BTS & 0.000 & 0.005 & 0.005 & 0.029 & 0.009 & 0.037 & 0.014 & 0.013 & 0.027 & 0.017 & 0.033 & 0.049 \\
\hline & & PRC & 0.000 & 0.028 & 0.028 & 0.000 & 0.052 & 0.052 & 0.000 & 0.094 & 0.094 & 0.001 & 0.070 & 0.071 \\
\hline & & $\mathrm{BC}$ & 0.000 & 0.065 & 0.065 & 0.001 & 0.057 & 0.057 & 0.014 & 0.063 & 0.077 & 0.057 & 0.028 & 0.085 \\
\hline & & BCA & 0.000 & 0.090 & 0.090 & 0.000 & 0.086 & 0.086 & 0.012 & 0.090 & 0.102 & 0.040 & 0.034 & 0.074 \\
\hline & & JAC & 0.000 & 0.024 & 0.024 & 0.029 & 0.016 & 0.045 & 0.014 & 0.033 & 0.046 & 0.037 & 0.020 & 0.057 \\
\hline & & BSD & 0.009 & 0.043 & 0.051 & 0.020 & 0.025 & 0.045 & 0.023 & 0.027 & 0.049 & 0.023 & 0.030 & 0.053 \\
\hline & \multirow[t]{7}{*}{0.6} & $\mathrm{CM}$ & 0.013 & 0.035 & 0.047 & 0.014 & 0.020 & 0.034 & 0.014 & 0.028 & 0.041 & 0.027 & 0.026 & 0.053 \\
\hline & & BTS & 0.013 & 0.016 & 0.028 & 0.014 & 0.014 & 0.028 & 0.014 & 0.011 & 0.025 & 0.026 & 0.021 & 0.047 \\
\hline & & PRC & 0.000 & 0.016 & 0.016 & 0.003 & 0.019 & 0.022 & 0.004 & 0.025 & 0.029 & 0.007 & 0.039 & 0.046 \\
\hline & & $\mathrm{BC}$ & 0.013 & 0.075 & 0.088 & 0.014 & 0.042 & 0.056 & 0.040 & 0.042 & 0.082 & 0.031 & 0.039 & 0.070 \\
\hline & & BCA & 0.000 & 0.075 & 0.075 & 0.014 & 0.045 & 0.059 & 0.040 & 0.046 & 0.086 & 0.027 & 0.045 & 0.072 \\
\hline & & JAC & 0.013 & 0.035 & 0.047 & 0.014 & 0.020 & 0.034 & 0.014 & 0.028 & 0.041 & 0.027 & 0.026 & 0.053 \\
\hline & & BSD & 0.020 & 0.041 & 0.061 & 0.018 & 0.027 & 0.045 & 0.016 & 0.032 & 0.048 & 0.019 & 0.030 & 0.049 \\
\hline & \multirow[t]{7}{*}{0.8} & $\mathrm{CM}$ & 0.024 & 0.066 & 0.090 & 0.018 & 0.028 & 0.046 & 0.017 & 0.029 & 0.045 & 0.024 & 0.036 & 0.060 \\
\hline & & BTS & 0.023 & 0.018 & 0.041 & 0.018 & 0.009 & 0.027 & 0.017 & 0.009 & 0.026 & 0.024 & 0.018 & 0.042 \\
\hline & & PRC & 0.004 & 0.018 & 0.022 & 0.004 & 0.027 & 0.031 & 0.010 & 0.017 & 0.026 & 0.019 & 0.018 & 0.037 \\
\hline & & BC & 0.005 & 0.104 & 0.109 & 0.006 & 0.097 & 0.103 & 0.016 & 0.094 & 0.109 & 0.033 & 0.060 & 0.092 \\
\hline & & BCA & 0.002 & 0.104 & 0.106 & 0.006 & 0.099 & 0.105 & 0.011 & 0.094 & 0.105 & 0.027 & 0.062 & 0.089 \\
\hline & & JAC & 0.024 & 0.066 & 0.090 & 0.018 & 0.028 & 0.046 & 0.017 & 0.029 & 0.045 & 0.024 & 0.036 & 0.060 \\
\hline & & BSD & 0.017 & 0.052 & 0.069 & 0.020 & 0.043 & 0.063 & 0.015 & 0.038 & 0.053 & 0.018 & 0.031 & 0.049 \\
\hline
\end{tabular}


Table 1 Continued:

\begin{tabular}{|c|c|c|c|c|c|c|c|c|c|c|c|c|c|c|}
\hline \multirow[t]{7}{*}{2} & \multirow[t]{7}{*}{0.4} & $\mathrm{CM}$ & 0.000 & 0.024 & 0.024 & 0.024 & 0.016 & 0.040 & 0.013 & 0.032 & 0.044 & 0.019 & 0.024 & 0.043 \\
\hline & & BTS & 0.000 & 0.006 & 0.006 & 0.024 & 0.011 & 0.034 & 0.013 & 0.012 & 0.025 & 0.019 & 0.038 & 0.057 \\
\hline & & PRC & 0.000 & 0.028 & 0.028 & 0.000 & 0.049 & 0.049 & 0.000 & 0.085 & 0.085 & 0.000 & 0.090 & 0.090 \\
\hline & & BC & 0.000 & 0.074 & 0.074 & 0.005 & 0.055 & 0.060 & 0.013 & 0.047 & 0.060 & 0.032 & 0.036 & 0.067 \\
\hline & & BCA & 0.000 & 0.084 & 0.084 & 0.002 & 0.100 & 0.102 & 0.009 & 0.066 & 0.075 & 0.021 & 0.045 & 0.066 \\
\hline & & JAC & 0.000 & 0.024 & 0.024 & 0.024 & 0.016 & 0.040 & 0.013 & 0.032 & 0.044 & 0.043 & 0.024 & 0.067 \\
\hline & & BSD & 0.007 & 0.048 & 0.054 & 0.016 & 0.032 & 0.048 & 0.026 & 0.030 & 0.056 & 0.022 & 0.025 & 0.046 \\
\hline & \multirow[t]{7}{*}{0.6} & $\mathrm{CM}$ & 0.015 & 0.024 & 0.039 & 0.011 & 0.015 & 0.026 & 0.020 & 0.033 & 0.053 & 0.024 & 0.020 & 0.044 \\
\hline & & BTS & 0.015 & 0.011 & 0.026 & 0.011 & 0.011 & 0.022 & 0.020 & 0.011 & 0.030 & 0.024 & 0.019 & 0.042 \\
\hline & & PRC & 0.000 & 0.011 & 0.011 & 0.001 & 0.013 & 0.013 & 0.007 & 0.031 & 0.038 & 0.007 & 0.034 & 0.041 \\
\hline & & $\mathrm{BC}$ & 0.015 & 0.078 & 0.093 & 0.011 & 0.046 & 0.057 & 0.068 & 0.058 & 0.126 & 0.039 & 0.037 & 0.076 \\
\hline & & BCA & 0.000 & 0.079 & 0.079 & 0.011 & 0.052 & 0.063 & 0.056 & 0.062 & 0.118 & 0.037 & 0.039 & 0.076 \\
\hline & & JAC & 0.015 & 0.024 & 0.039 & 0.011 & 0.015 & 0.026 & 0.020 & 0.033 & 0.053 & 0.024 & 0.020 & 0.044 \\
\hline & & BSD & 0.018 & 0.034 & 0.051 & 0.018 & 0.027 & 0.045 & 0.022 & 0.032 & 0.054 & 0.022 & 0.028 & 0.050 \\
\hline & \multirow[t]{7}{*}{0.8} & $\mathrm{CM}$ & 0.023 & 0.067 & 0.090 & 0.024 & 0.032 & 0.056 & 0.015 & 0.037 & 0.052 & 0.020 & 0.034 & 0.054 \\
\hline & & BTS & 0.022 & 0.020 & 0.042 & 0.024 & 0.007 & 0.030 & 0.016 & 0.012 & 0.028 & 0.020 & 0.019 & 0.038 \\
\hline & & PRC & 0.005 & 0.020 & 0.025 & 0.005 & 0.032 & 0.037 & 0.007 & 0.022 & 0.029 & 0.017 & 0.019 & 0.035 \\
\hline & & BC & 0.008 & 0.127 & 0.135 & 0.005 & 0.133 & 0.138 & 0.017 & 0.094 & 0.111 & 0.023 & 0.049 & 0.072 \\
\hline & & BCA & 0.005 & 0.128 & 0.132 & 0.005 & 0.134 & 0.139 & 0.011 & 0.094 & 0.105 & 0.022 & 0.050 & 0.072 \\
\hline & & JAC & 0.023 & 0.067 & 0.090 & 0.024 & 0.032 & 0.056 & 0.015 & 0.037 & 0.052 & 0.020 & 0.034 & 0.054 \\
\hline & & BSD & 0.018 & 0.053 & 0.070 & 0.027 & 0.049 & 0.075 & 0.020 & 0.041 & 0.061 & 0.018 & 0.031 & 0.048 \\
\hline
\end{tabular}

Table 2: 95\% confidence interval for $\theta$ based on $\mathrm{k}=4$.

\begin{tabular}{|c|c|c|c|c|c|c|c|c|c|c|c|c|c|c|}
\hline & & $\mathrm{n}$ & & 20 & & & 30 & & & 50 & & & 100 & \\
\hline $\mathrm{R}$ & $\mathrm{CP}$ & Method & $\mathrm{L}$ & $\mathrm{U}$ & $\mathrm{T}$ & $\mathrm{L}$ & $\mathrm{U}$ & $\mathrm{T}$ & $\mathrm{L}$ & $\mathrm{U}$ & $\mathrm{T}$ & $\mathrm{L}$ & $\mathrm{U}$ & $\mathrm{T}$ \\
\hline \multirow[t]{14}{*}{0.4} & \multirow[t]{7}{*}{0.4} & CM & 0.000 & 0.036 & 0.036 & 0.005 & 0.026 & 0.031 & 0.009 & 0.025 & 0.034 & 0.014 & 0.023 & 0.037 \\
\hline & & BTS & 0.000 & 0.016 & 0.016 & 0.001 & 0.024 & 0.025 & 0.003 & 0.053 & 0.056 & 0.003 & 0.072 & 0.075 \\
\hline & & PRC & 0.000 & 0.067 & 0.067 & 0.000 & 0.078 & 0.078 & 0.000 & 0.097 & 0.097 & 0.001 & 0.104 & 0.104 \\
\hline & & BC & 0.014 & 0.035 & 0.049 & 0.032 & 0.019 & 0.051 & 0.048 & 0.013 & 0.061 & 0.069 & 0.010 & 0.078 \\
\hline & & BCA & 0.007 & 0.045 & 0.051 & 0.023 & 0.028 & 0.051 & 0.043 & 0.020 & 0.063 & 0.062 & 0.011 & 0.073 \\
\hline & & JAC & 0.000 & 0.039 & 0.039 & 0.005 & 0.029 & 0.033 & 0.011 & 0.028 & 0.039 & 0.015 & 0.024 & 0.039 \\
\hline & & BSD & 0.002 & 0.037 & 0.039 & 0.007 & 0.030 & 0.037 & 0.010 & 0.026 & 0.036 & 0.016 & 0.024 & 0.040 \\
\hline & \multirow[t]{7}{*}{0.6} & $\mathrm{CM}$ & 0.007 & 0.046 & 0.053 & 0.008 & 0.044 & 0.052 & 0.013 & 0.037 & 0.050 & 0.024 & 0.024 & 0.048 \\
\hline & & BTS & 0.002 & 0.021 & 0.022 & 0.004 & 0.030 & 0.033 & 0.005 & 0.038 & 0.043 & 0.010 & 0.042 & 0.052 \\
\hline & & PRC & 0.000 & 0.046 & 0.046 & 0.000 & 0.069 & 0.069 & 0.001 & 0.057 & 0.058 & 0.002 & 0.057 & 0.058 \\
\hline & & BC & 0.022 & 0.052 & 0.074 & 0.023 & 0.056 & 0.079 & 0.035 & 0.033 & 0.068 & 0.051 & 0.017 & 0.068 \\
\hline & & BCA & 0.012 & 0.055 & 0.067 & 0.017 & 0.063 & 0.079 & 0.029 & 0.038 & 0.067 & 0.046 & 0.019 & 0.065 \\
\hline & & JAC & 0.008 & 0.047 & 0.055 & 0.009 & 0.045 & 0.053 & 0.013 & 0.041 & 0.054 & 0.024 & 0.024 & 0.048 \\
\hline & & BSD & 0.006 & 0.047 & 0.053 & 0.009 & 0.045 & 0.053 & 0.013 & 0.041 & 0.054 & 0.022 & 0.028 & 0.050 \\
\hline & \multirow[t]{7}{*}{0.8} & $\mathrm{CM}$ & 0.008 & 0.057 & 0.065 & 0.012 & 0.042 & 0.054 & 0.014 & 0.039 & 0.053 & 0.019 & 0.031 & 0.050 \\
\hline & & BTS & 0.005 & 0.022 & 0.026 & 0.007 & 0.021 & 0.027 & 0.008 & 0.025 & 0.033 & 0.009 & 0.025 & 0.033 \\
\hline & & PRC & 0.001 & 0.027 & 0.028 & 0.002 & 0.030 & 0.031 & 0.003 & 0.028 & 0.031 & 0.006 & 0.028 & 0.034 \\
\hline & & BC & 0.014 & 0.092 & 0.106 & 0.014 & 0.072 & 0.086 & 0.021 & 0.050 & 0.071 & 0.026 & 0.040 & 0.065 \\
\hline & & BCA & 0.010 & 0.095 & 0.105 & 0.012 & 0.073 & 0.085 & 0.019 & 0.051 & 0.069 & 0.025 & 0.042 & 0.066 \\
\hline & & JAC & 0.011 & 0.057 & 0.068 & 0.012 & 0.043 & 0.054 & 0.015 & 0.040 & 0.055 & 0.020 & 0.031 & 0.051 \\
\hline & & BSD & 0.011 & 0.059 & 0.070 & 0.012 & 0.046 & 0.057 & 0.014 & 0.040 & 0.054 & 0.018 & 0.031 & 0.049 \\
\hline
\end{tabular}


Table 2: Continued

\begin{tabular}{|c|c|c|c|c|c|c|c|c|c|c|c|c|c|c|}
\hline \multirow[t]{21}{*}{0.8} & \multirow[t]{7}{*}{0.4} & $\mathrm{CM}$ & 0.000 & 0.036 & 0.036 & 0.004 & 0.027 & 0.031 & 0.009 & 0.029 & 0.038 & 0.015 & 0.027 & 0.042 \\
\hline & & BTS & 0.000 & 0.016 & 0.016 & 0.002 & 0.027 & 0.029 & 0.002 & 0.052 & 0.054 & 0.003 & 0.089 & 0.091 \\
\hline & & PRC & 0.000 & 0.065 & 0.065 & 0.000 & 0.073 & 0.073 & 0.000 & 0.101 & 0.101 & 0.000 & 0.119 & 0.119 \\
\hline & & BC & 0.015 & 0.035 & 0.050 & 0.032 & 0.021 & 0.052 & 0.046 & 0.020 & 0.065 & 0.090 & 0.008 & 0.098 \\
\hline & & BCA & 0.006 & 0.043 & 0.048 & 0.025 & 0.028 & 0.053 & 0.043 & 0.024 & 0.067 & 0.082 & 0.013 & 0.095 \\
\hline & & JAC & 0.000 & 0.038 & 0.038 & 0.004 & 0.028 & 0.032 & 0.010 & 0.032 & 0.042 & 0.016 & 0.028 & 0.044 \\
\hline & & BSD & 0.003 & 0.036 & 0.039 & 0.009 & 0.027 & 0.036 & 0.012 & 0.030 & 0.041 & 0.017 & 0.026 & 0.043 \\
\hline & \multirow[t]{7}{*}{0.6} & $\mathrm{CM}$ & 0.005 & 0.039 & 0.044 & 0.009 & 0.034 & 0.043 & 0.013 & 0.027 & 0.040 & 0.015 & 0.030 & 0.045 \\
\hline & & BTS & 0.001 & 0.016 & 0.017 & 0.003 & 0.021 & 0.024 & 0.005 & 0.026 & 0.031 & 0.004 & 0.048 & 0.051 \\
\hline & & PRC & 0.000 & 0.041 & 0.041 & 0.000 & 0.068 & 0.068 & 0.001 & 0.044 & 0.045 & 0.000 & 0.065 & 0.065 \\
\hline & & BC & 0.019 & 0.042 & 0.061 & 0.018 & 0.053 & 0.071 & 0.035 & 0.025 & 0.059 & 0.049 & 0.021 & 0.070 \\
\hline & & BCA & 0.010 & 0.047 & 0.057 & 0.015 & 0.060 & 0.075 & 0.028 & 0.029 & 0.057 & 0.044 & 0.026 & 0.070 \\
\hline & & JAC & 0.009 & 0.039 & 0.048 & 0.010 & 0.037 & 0.046 & 0.013 & 0.029 & 0.042 & 0.015 & 0.030 & 0.045 \\
\hline & & BSD & 0.007 & 0.040 & 0.046 & 0.008 & 0.037 & 0.044 & 0.015 & 0.031 & 0.045 & 0.018 & 0.031 & 0.048 \\
\hline & \multirow[t]{7}{*}{0.8} & $\mathrm{CM}$ & 0.008 & 0.051 & 0.059 & 0.016 & 0.046 & 0.062 & 0.016 & 0.037 & 0.053 & 0.019 & 0.034 & 0.053 \\
\hline & & BTS & 0.004 & 0.014 & 0.018 & 0.008 & 0.021 & 0.029 & 0.010 & 0.023 & 0.032 & 0.009 & 0.028 & 0.036 \\
\hline & & PRC & 0.000 & 0.024 & 0.024 & 0.002 & 0.030 & 0.032 & 0.005 & 0.026 & 0.031 & 0.006 & 0.031 & 0.037 \\
\hline & & BC & 0.011 & 0.089 & 0.100 & 0.020 & 0.069 & 0.088 & 0.024 & 0.048 & 0.072 & 0.026 & 0.043 & 0.069 \\
\hline & & BCA & 0.010 & 0.089 & 0.098 & 0.018 & 0.069 & 0.087 & 0.021 & 0.048 & 0.069 & 0.024 & 0.044 & 0.068 \\
\hline & & JAC & 0.010 & 0.051 & 0.061 & 0.018 & 0.046 & 0.064 & 0.017 & 0.038 & 0.054 & 0.020 & 0.034 & 0.054 \\
\hline & & BSD & 0.009 & 0.056 & 0.065 & 0.019 & 0.045 & 0.064 & 0.016 & 0.039 & 0.054 & 0.015 & 0.037 & 0.051 \\
\hline \multirow[t]{21}{*}{1.2} & \multirow[t]{7}{*}{0.4} & $\mathrm{CM}$ & 0.000 & 0.029 & 0.029 & 0.004 & 0.025 & 0.028 & 0.011 & 0.027 & 0.038 & 0.020 & 0.023 & 0.043 \\
\hline & & BTS & 0.000 & 0.012 & 0.012 & 0.001 & 0.027 & 0.028 & 0.002 & 0.049 & 0.051 & 0.004 & 0.088 & 0.092 \\
\hline & & PRC & 0.000 & 0.064 & 0.064 & 0.000 & 0.074 & 0.074 & 0.000 & 0.097 & 0.097 & 0.000 & 0.112 & 0.112 \\
\hline & & BC & 0.004 & 0.028 & 0.032 & 0.026 & 0.018 & 0.044 & 0.051 & 0.017 & 0.068 & 0.094 & 0.010 & 0.104 \\
\hline & & BCA & 0.000 & 0.038 & 0.038 & 0.011 & 0.022 & 0.033 & 0.041 & 0.022 & 0.062 & 0.087 & 0.012 & 0.099 \\
\hline & & JAC & 0.000 & 0.032 & 0.032 & 0.004 & 0.026 & 0.030 & 0.014 & 0.030 & 0.043 & 0.021 & 0.023 & 0.044 \\
\hline & & BSD & 0.002 & 0.034 & 0.036 & 0.004 & 0.029 & 0.033 & 0.013 & 0.033 & 0.046 & 0.019 & 0.024 & 0.043 \\
\hline & \multirow[t]{7}{*}{0.6} & $\mathrm{CM}$ & 0.007 & 0.049 & 0.056 & 0.011 & 0.036 & 0.047 & 0.012 & 0.027 & 0.039 & 0.018 & 0.034 & 0.052 \\
\hline & & BTS & 0.004 & 0.023 & 0.026 & 0.005 & 0.027 & 0.032 & 0.003 & 0.027 & 0.030 & 0.004 & 0.048 & 0.051 \\
\hline & & PRC & 0.000 & 0.054 & 0.054 & 0.000 & 0.064 & 0.064 & 0.000 & 0.047 & 0.047 & 0.001 & 0.059 & 0.060 \\
\hline & & BC & 0.013 & 0.060 & 0.073 & 0.019 & 0.048 & 0.066 & 0.029 & 0.022 & 0.051 & 0.047 & 0.021 & 0.068 \\
\hline & & BCA & 0.008 & 0.067 & 0.074 & 0.013 & 0.051 & 0.063 & 0.024 & 0.029 & 0.053 & 0.041 & 0.024 & 0.065 \\
\hline & & JAC & 0.009 & 0.050 & 0.058 & 0.011 & 0.039 & 0.050 & 0.012 & 0.027 & 0.039 & 0.018 & 0.034 & 0.052 \\
\hline & & BSD & 0.009 & 0.052 & 0.061 & 0.012 & 0.039 & 0.051 & 0.012 & 0.029 & 0.041 & 0.016 & 0.033 & 0.049 \\
\hline & \multirow[t]{7}{*}{0.8} & $\mathrm{CM}$ & 0.009 & 0.050 & 0.059 & 0.012 & 0.043 & 0.054 & 0.009 & 0.043 & 0.052 & 0.015 & 0.029 & 0.044 \\
\hline & & BTS & 0.006 & 0.016 & 0.022 & 0.004 & 0.019 & 0.022 & 0.004 & 0.024 & 0.027 & 0.007 & 0.023 & 0.030 \\
\hline & & PRC & 0.000 & 0.024 & 0.024 & 0.001 & 0.027 & 0.027 & 0.002 & 0.027 & 0.028 & 0.004 & 0.026 & 0.030 \\
\hline & & BC & 0.014 & 0.096 & 0.110 & 0.013 & 0.074 & 0.086 & 0.018 & 0.057 & 0.075 & 0.021 & 0.038 & 0.059 \\
\hline & & BCA & 0.010 & 0.095 & 0.104 & 0.010 & 0.075 & 0.085 & 0.015 & 0.060 & 0.074 & 0.021 & 0.040 & 0.061 \\
\hline & & JAC & 0.012 & 0.050 & 0.062 & 0.012 & 0.043 & 0.055 & 0.010 & 0.044 & 0.054 & 0.015 & 0.029 & 0.044 \\
\hline & & BSD & 0.012 & 0.053 & 0.065 & 0.007 & 0.046 & 0.053 & 0.009 & 0.042 & 0.051 & 0.015 & 0.033 & 0.048 \\
\hline
\end{tabular}


Table 2: Continued

\begin{tabular}{|c|c|c|c|c|c|c|c|c|c|c|c|c|c|c|}
\hline \multirow[t]{7}{*}{1.6} & \multirow[t]{7}{*}{0.4} & CM & 0.000 & 0.033 & 0.033 & 0.009 & 0.029 & 0.038 & 0.011 & 0.029 & 0.039 & 0.018 & 0.025 & 0.043 \\
\hline & & BTS & 0.000 & 0.014 & 0.014 & 0.003 & 0.032 & 0.035 & 0.003 & 0.049 & 0.052 & 0.002 & 0.099 & 0.101 \\
\hline & & PRC & 0.000 & 0.060 & 0.060 & 0.000 & 0.082 & 0.082 & 0.000 & 0.088 & 0.088 & 0.000 & 0.135 & 0.135 \\
\hline & & BC & 0.011 & 0.033 & 0.044 & 0.040 & 0.021 & 0.061 & 0.054 & 0.020 & 0.074 & 0.080 & 0.011 & 0.090 \\
\hline & & BCA & 0.006 & 0.040 & 0.046 & 0.027 & 0.030 & 0.057 & 0.044 & 0.024 & 0.068 & 0.075 & 0.014 & 0.089 \\
\hline & & JAC & 0.000 & 0.034 & 0.034 & 0.009 & 0.033 & 0.042 & 0.012 & 0.031 & 0.043 & 0.019 & 0.025 & 0.044 \\
\hline & & BSD & 0.004 & 0.034 & 0.038 & 0.010 & 0.035 & 0.045 & 0.009 & 0.035 & 0.043 & 0.016 & 0.027 & 0.043 \\
\hline & \multirow[t]{7}{*}{0.6} & CM & 0.007 & 0.045 & 0.051 & 0.007 & 0.032 & 0.039 & 0.014 & 0.032 & 0.046 & 0.019 & 0.028 & 0.047 \\
\hline & & BTS & 0.003 & 0.019 & 0.022 & 0.003 & 0.022 & 0.025 & 0.003 & 0.030 & 0.033 & 0.005 & 0.046 & 0.051 \\
\hline & & PRC & 0.000 & 0.047 & 0.047 & 0.000 & 0.072 & 0.072 & 0.000 & 0.053 & 0.053 & 0.001 & 0.065 & 0.065 \\
\hline & & BC & 0.020 & 0.051 & 0.071 & 0.019 & 0.054 & 0.073 & 0.039 & 0.027 & 0.066 & 0.046 & 0.018 & 0.064 \\
\hline & & BCA & 0.011 & 0.059 & 0.070 & 0.014 & 0.059 & 0.073 & 0.033 & 0.032 & 0.065 & 0.044 & 0.023 & 0.067 \\
\hline & & JAC & 0.010 & 0.047 & 0.057 & 0.007 & 0.038 & 0.045 & 0.014 & 0.034 & 0.048 & 0.019 & 0.028 & 0.047 \\
\hline & & BSD & 0.010 & 0.046 & 0.056 & 0.006 & 0.037 & 0.042 & 0.014 & 0.030 & 0.044 & 0.015 & 0.032 & 0.047 \\
\hline & \multirow[t]{7}{*}{0.8} & CM & 0.008 & 0.056 & 0.063 & 0.011 & 0.045 & 0.056 & 0.014 & 0.034 & 0.048 & 0.024 & 0.032 & 0.056 \\
\hline & & BTS & 0.005 & 0.020 & 0.025 & 0.005 & 0.022 & 0.026 & 0.006 & 0.017 & 0.023 & 0.015 & 0.021 & 0.036 \\
\hline & & PRC & 0.001 & 0.026 & 0.027 & 0.002 & 0.031 & 0.032 & 0.002 & 0.018 & 0.020 & 0.010 & 0.025 & 0.034 \\
\hline & & BC & 0.010 & 0.093 & 0.103 & 0.014 & 0.077 & 0.090 & 0.021 & 0.045 & 0.065 & 0.032 & 0.038 & 0.070 \\
\hline & & BCA & 0.009 & 0.094 & 0.102 & 0.009 & 0.077 & 0.086 & 0.019 & 0.047 & 0.066 & 0.030 & 0.039 & 0.069 \\
\hline & & JAC & 0.012 & 0.056 & 0.068 & 0.012 & 0.046 & 0.057 & 0.016 & 0.034 & 0.050 & 0.024 & 0.033 & 0.057 \\
\hline & & BSD & 0.009 & 0.058 & 0.067 & 0.009 & 0.047 & 0.056 & 0.012 & 0.038 & 0.049 & 0.021 & 0.030 & 0.050 \\
\hline \multirow[t]{14}{*}{2} & \multirow[t]{7}{*}{0.4} & CM & 0.000 & 0.031 & 0.031 & 0.004 & 0.026 & 0.030 & 0.011 & 0.021 & 0.032 & 0.018 & 0.023 & 0.041 \\
\hline & & BTS & 0.000 & 0.015 & 0.015 & 0.002 & 0.030 & 0.031 & 0.003 & 0.051 & 0.054 & 0.004 & 0.090 & 0.094 \\
\hline & & PRC & 0.000 & 0.063 & 0.063 & 0.000 & 0.075 & 0.075 & 0.000 & 0.094 & 0.094 & 0.000 & 0.115 & 0.115 \\
\hline & & $\mathrm{BC}$ & 0.005 & 0.031 & 0.036 & 0.033 & 0.023 & 0.056 & 0.055 & 0.011 & 0.066 & 0.079 & 0.010 & 0.089 \\
\hline & & BCA & 0.000 & 0.041 & 0.041 & 0.021 & 0.029 & 0.050 & 0.050 & 0.015 & 0.065 & 0.073 & 0.014 & 0.087 \\
\hline & & JAC & 0.000 & 0.035 & 0.035 & 0.004 & 0.031 & 0.035 & 0.014 & 0.023 & 0.037 & 0.020 & 0.024 & 0.044 \\
\hline & & BSD & 0.004 & 0.038 & 0.042 & 0.009 & 0.029 & 0.038 & 0.012 & 0.026 & 0.038 & 0.019 & 0.026 & 0.045 \\
\hline & \multirow[t]{7}{*}{0.6} & CM & 0.005 & 0.042 & 0.047 & 0.007 & 0.041 & 0.047 & 0.016 & 0.038 & 0.053 & 0.017 & 0.032 & 0.049 \\
\hline & & BTS & 0.002 & 0.014 & 0.016 & 0.003 & 0.028 & 0.031 & 0.003 & 0.037 & 0.040 & 0.007 & 0.053 & 0.060 \\
\hline & & PRC & 0.000 & 0.044 & 0.044 & 0.000 & 0.074 & 0.074 & 0.000 & 0.058 & 0.058 & 0.001 & 0.071 & 0.072 \\
\hline & & $\mathrm{BC}$ & 0.012 & 0.052 & 0.064 & 0.018 & 0.055 & 0.073 & 0.034 & 0.032 & 0.065 & 0.048 & 0.024 & 0.072 \\
\hline & & BCA & 0.006 & 0.057 & 0.063 & 0.012 & 0.068 & 0.079 & 0.025 & 0.038 & 0.063 & 0.043 & 0.027 & 0.069 \\
\hline & & JAC & 0.008 & 0.043 & 0.050 & 0.007 & 0.043 & 0.050 & 0.016 & 0.041 & 0.057 & 0.017 & 0.033 & 0.050 \\
\hline & & BSD & 0.007 & 0.046 & 0.053 & 0.009 & 0.043 & 0.052 & 0.013 & 0.036 & 0.049 & 0.016 & 0.035 & 0.051 \\
\hline & \multirow[t]{7}{*}{0.8} & CM & 0.008 & 0.053 & 0.061 & 0.013 & 0.043 & 0.056 & 0.010 & 0.038 & 0.047 & 0.020 & 0.030 & 0.050 \\
\hline & & BTS & 0.006 & 0.020 & 0.026 & 0.006 & 0.022 & 0.027 & 0.004 & 0.022 & 0.026 & 0.013 & 0.022 & 0.035 \\
\hline & & PRC & 0.001 & 0.025 & 0.025 & 0.001 & 0.030 & 0.030 & 0.001 & 0.024 & 0.025 & 0.009 & 0.025 & 0.034 \\
\hline & & $\mathrm{BC}$ & 0.012 & 0.088 & 0.099 & 0.015 & 0.064 & 0.079 & 0.016 & 0.053 & 0.069 & 0.027 & 0.044 & 0.070 \\
\hline & & BCA & 0.008 & 0.088 & 0.095 & 0.012 & 0.065 & 0.077 & 0.013 & 0.055 & 0.068 & 0.025 & 0.045 & 0.070 \\
\hline & & JAC & 0.010 & 0.053 & 0.063 & 0.014 & 0.043 & 0.056 & 0.010 & 0.039 & 0.049 & 0.020 & 0.031 & 0.051 \\
\hline & & BSD & 0.009 & 0.053 & 0.062 & 0.011 & 0.042 & 0.053 & 0.010 & 0.041 & 0.051 & 0.020 & 0.032 & 0.052 \\
\hline
\end{tabular}


Table 3: 95\% confidence interval for $\theta$ based on $\mathrm{k}=8$.

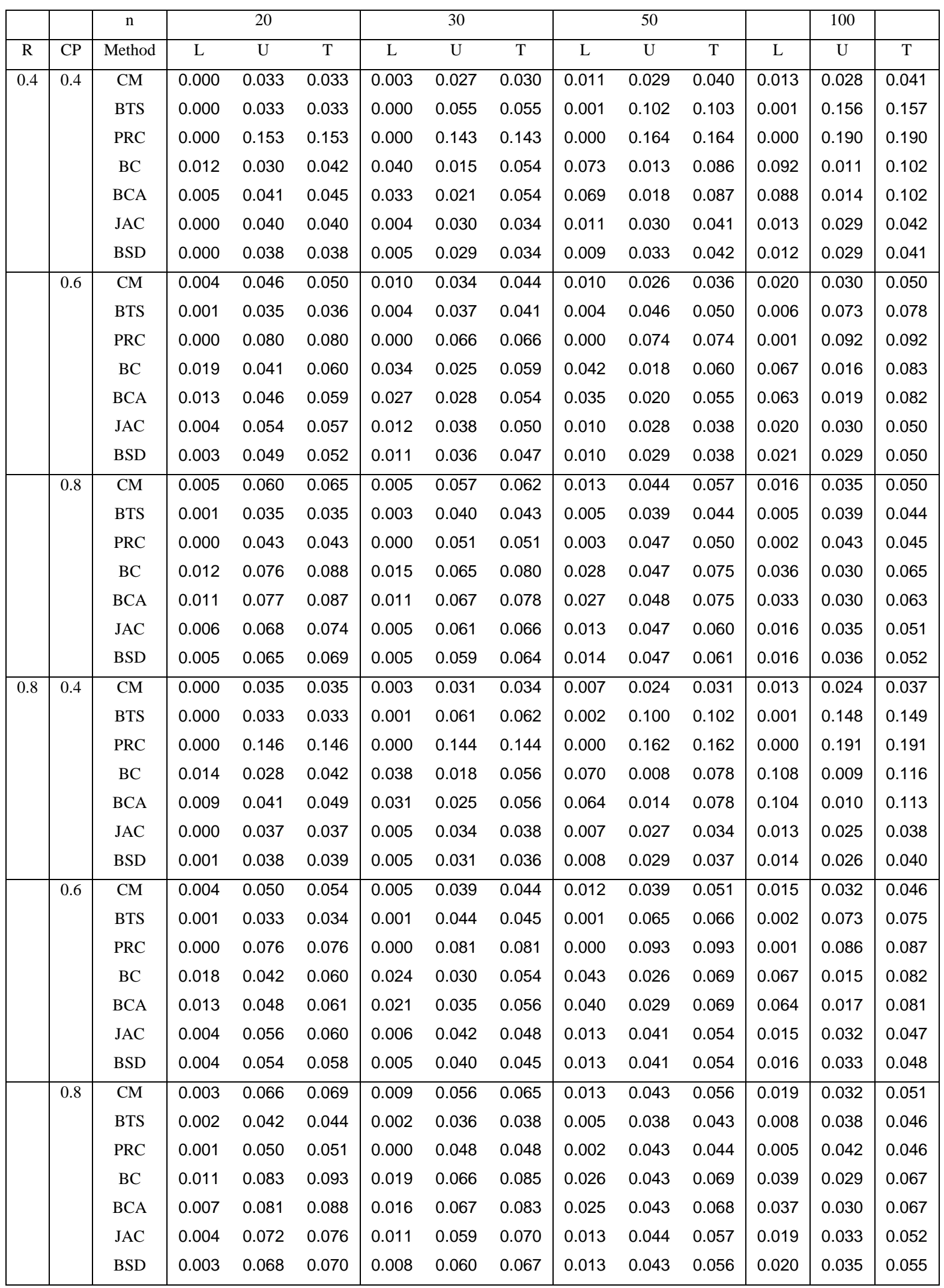


Table 3: Continued

\begin{tabular}{|c|c|c|c|c|c|c|c|c|c|c|c|c|c|c|}
\hline \multirow[t]{7}{*}{1.2} & \multirow[t]{7}{*}{0.4} & $\mathrm{CM}$ & 0.000 & 0.036 & 0.036 & 0.004 & 0.038 & 0.041 & 0.011 & 0.026 & 0.037 & 0.014 & 0.027 & 0.041 \\
\hline & & BTS & 0.000 & 0.036 & 0.036 & 0.001 & 0.059 & 0.059 & 0.001 & 0.098 & 0.099 & 0.001 & 0.147 & 0.148 \\
\hline & & PRC & 0.000 & 0.148 & 0.148 & 0.000 & 0.144 & 0.144 & 0.000 & 0.156 & 0.156 & 0.000 & 0.184 & 0.184 \\
\hline & & BC & 0.013 & 0.034 & 0.047 & 0.040 & 0.025 & 0.065 & 0.059 & 0.010 & 0.069 & 0.099 & 0.011 & 0.110 \\
\hline & & BCA & 0.005 & 0.042 & 0.047 & 0.033 & 0.030 & 0.063 & 0.057 & 0.015 & 0.071 & 0.096 & 0.016 & 0.111 \\
\hline & & JAC & 0.000 & 0.040 & 0.040 & 0.004 & 0.041 & 0.045 & 0.011 & 0.029 & 0.040 & 0.014 & 0.031 & 0.045 \\
\hline & & BSD & 0.001 & 0.042 & 0.043 & 0.005 & 0.039 & 0.044 & 0.011 & 0.029 & 0.039 & 0.015 & 0.028 & 0.043 \\
\hline & \multirow[t]{7}{*}{0.6} & $\mathrm{CM}$ & 0.007 & 0.052 & 0.059 & 0.008 & 0.050 & 0.058 & 0.012 & 0.038 & 0.050 & 0.016 & 0.031 & 0.046 \\
\hline & & BTS & 0.001 & 0.040 & 0.041 & 0.001 & 0.055 & 0.056 & 0.002 & 0.061 & 0.063 & 0.001 & 0.081 & 0.081 \\
\hline & & PRC & 0.000 & 0.070 & 0.070 & 0.000 & 0.086 & 0.086 & 0.000 & 0.084 & 0.084 & 0.000 & 0.099 & 0.099 \\
\hline & & $\mathrm{BC}$ & 0.019 & 0.047 & 0.066 & 0.032 & 0.038 & 0.069 & 0.045 & 0.026 & 0.071 & 0.069 & 0.016 & 0.084 \\
\hline & & BCA & 0.014 & 0.051 & 0.065 & 0.025 & 0.042 & 0.066 & 0.040 & 0.030 & 0.069 & 0.067 & 0.017 & 0.084 \\
\hline & & JAC & 0.007 & 0.056 & 0.063 & 0.008 & 0.054 & 0.061 & 0.012 & 0.040 & 0.052 & 0.016 & 0.032 & 0.047 \\
\hline & & BSD & 0.007 & 0.054 & 0.061 & 0.007 & 0.051 & 0.057 & 0.012 & 0.041 & 0.053 & 0.015 & 0.035 & 0.049 \\
\hline & \multirow[t]{7}{*}{0.8} & $\mathrm{CM}$ & 0.008 & 0.055 & 0.063 & 0.010 & 0.051 & 0.061 & 0.016 & 0.044 & 0.060 & 0.017 & 0.038 & 0.055 \\
\hline & & BTS & 0.004 & 0.032 & 0.036 & 0.002 & 0.038 & 0.040 & 0.008 & 0.038 & 0.045 & 0.006 & 0.041 & 0.047 \\
\hline & & PRC & 0.001 & 0.042 & 0.043 & 0.000 & 0.048 & 0.048 & 0.002 & 0.043 & 0.045 & 0.004 & 0.047 & 0.051 \\
\hline & & $\mathrm{BC}$ & 0.014 & 0.074 & 0.088 & 0.020 & 0.065 & 0.085 & 0.031 & 0.044 & 0.075 & 0.031 & 0.032 & 0.063 \\
\hline & & BCA & 0.011 & 0.073 & 0.084 & 0.016 & 0.064 & 0.080 & 0.029 & 0.044 & 0.073 & 0.029 & 0.033 & 0.062 \\
\hline & & JAC & 0.010 & 0.059 & 0.068 & 0.011 & 0.057 & 0.068 & 0.017 & 0.045 & 0.062 & 0.018 & 0.038 & 0.055 \\
\hline & & BSD & 0.009 & 0.058 & 0.066 & 0.010 & 0.054 & 0.064 & 0.016 & 0.046 & 0.062 & 0.019 & 0.037 & 0.055 \\
\hline \multirow[t]{14}{*}{1.6} & \multirow[t]{7}{*}{0.4} & $\mathrm{CM}$ & 0.000 & 0.033 & 0.033 & 0.003 & 0.030 & 0.033 & 0.012 & 0.027 & 0.038 & 0.017 & 0.026 & 0.043 \\
\hline & & BTS & 0.000 & 0.030 & 0.030 & 0.000 & 0.055 & 0.055 & 0.002 & 0.093 & 0.095 & 0.002 & 0.160 & 0.162 \\
\hline & & PRC & 0.000 & 0.141 & 0.141 & 0.000 & 0.140 & 0.140 & 0.000 & 0.155 & 0.155 & 0.000 & 0.192 & 0.192 \\
\hline & & $\mathrm{BC}$ & 0.005 & 0.031 & 0.036 & 0.036 & 0.018 & 0.053 & 0.075 & 0.014 & 0.089 & 0.112 & 0.010 & 0.121 \\
\hline & & BCA & 0.002 & 0.042 & 0.044 & 0.030 & 0.024 & 0.054 & 0.071 & 0.017 & 0.087 & 0.109 & 0.012 & 0.121 \\
\hline & & JAC & 0.000 & 0.043 & 0.043 & 0.004 & 0.032 & 0.035 & 0.012 & 0.028 & 0.039 & 0.017 & 0.026 & 0.043 \\
\hline & & BSD & 0.000 & 0.036 & 0.036 & 0.005 & 0.033 & 0.037 & 0.012 & 0.028 & 0.040 & 0.017 & 0.026 & 0.043 \\
\hline & \multirow[t]{7}{*}{0.6} & $\mathrm{CM}$ & 0.004 & 0.045 & 0.049 & 0.008 & 0.038 & 0.045 & 0.010 & 0.038 & 0.048 & 0.019 & 0.030 & 0.049 \\
\hline & & BTS & 0.000 & 0.032 & 0.032 & 0.003 & 0.043 & 0.046 & 0.002 & 0.063 & 0.065 & 0.004 & 0.074 & 0.078 \\
\hline & & PRC & 0.000 & 0.067 & 0.067 & 0.000 & 0.076 & 0.076 & 0.000 & 0.081 & 0.081 & 0.000 & 0.091 & 0.091 \\
\hline & & $\mathrm{BC}$ & 0.014 & 0.038 & 0.052 & 0.030 & 0.029 & 0.059 & 0.039 & 0.027 & 0.066 & 0.066 & 0.017 & 0.082 \\
\hline & & BCA & 0.009 & 0.044 & 0.052 & 0.022 & 0.031 & 0.053 & 0.037 & 0.030 & 0.066 & 0.063 & 0.019 & 0.081 \\
\hline & & JAC & 0.004 & 0.048 & 0.052 & 0.009 & 0.042 & 0.051 & 0.010 & 0.040 & 0.050 & 0.020 & 0.031 & 0.050 \\
\hline & & BSD & 0.003 & 0.046 & 0.049 & 0.007 & 0.036 & 0.043 & 0.009 & 0.040 & 0.049 & 0.018 & 0.032 & 0.050 \\
\hline & \multirow[t]{7}{*}{0.8} & CM & 0.007 & 0.055 & 0.062 & 0.013 & 0.048 & 0.060 & 0.013 & 0.036 & 0.049 & 0.019 & 0.032 & 0.051 \\
\hline & & BTS & 0.002 & 0.026 & 0.028 & 0.005 & 0.026 & 0.031 & 0.004 & 0.033 & 0.037 & 0.006 & 0.039 & 0.045 \\
\hline & & PRC & 0.000 & 0.036 & 0.036 & 0.001 & 0.042 & 0.042 & 0.001 & 0.036 & 0.037 & 0.003 & 0.045 & 0.048 \\
\hline & & $\mathrm{BC}$ & 0.014 & 0.069 & 0.082 & 0.023 & 0.056 & 0.079 & 0.026 & 0.038 & 0.063 & 0.038 & 0.028 & 0.066 \\
\hline & & BCA & 0.011 & 0.068 & 0.079 & 0.021 & 0.055 & 0.076 & 0.023 & 0.037 & 0.060 & 0.037 & 0.029 & 0.066 \\
\hline & & JAC & 0.008 & 0.060 & 0.068 & 0.013 & 0.050 & 0.063 & 0.013 & 0.038 & 0.051 & 0.020 & 0.033 & 0.053 \\
\hline & & BSD & 0.007 & 0.056 & 0.063 & 0.012 & 0.048 & 0.060 & 0.013 & 0.038 & 0.051 & 0.017 & 0.033 & 0.050 \\
\hline
\end{tabular}


Table 3: Continued

\begin{tabular}{|c|c|c|ccc|ccc|ccc|c|c|c|}
\hline 2 & 0.4 & CM & 0.000 & 0.031 & 0.031 & 0.007 & 0.031 & 0.038 & 0.006 & 0.029 & 0.034 & 0.017 & 0.034 & 0.051 \\
& & BTS & 0.000 & 0.032 & 0.032 & 0.002 & 0.060 & 0.062 & 0.001 & 0.102 & 0.103 & 0.000 & 0.147 & 0.147 \\
& & PRC & 0.000 & 0.133 & 0.133 & 0.000 & 0.143 & 0.143 & 0.000 & 0.158 & 0.158 & 0.000 & 0.178 & 0.178 \\
& & BC & 0.006 & 0.031 & 0.037 & 0.042 & 0.021 & 0.063 & 0.064 & 0.013 & 0.077 & 0.113 & 0.011 & 0.123 \\
& & BCA & 0.004 & 0.038 & 0.041 & 0.033 & 0.027 & 0.060 & 0.059 & 0.017 & 0.076 & 0.107 & 0.015 & 0.122 \\
& & JAC & 0.000 & 0.039 & 0.039 & 0.007 & 0.035 & 0.041 & 0.006 & 0.029 & 0.035 & 0.017 & 0.035 & 0.052 \\
& & BSD & 0.000 & 0.038 & 0.038 & 0.008 & 0.031 & 0.039 & 0.007 & 0.028 & 0.035 & 0.016 & 0.034 & 0.050 \\
\hline \multirow{3}{*}{0.6} & CM & 0.003 & 0.045 & 0.048 & 0.009 & 0.045 & 0.054 & 0.009 & 0.035 & 0.044 & 0.015 & 0.032 & 0.047 \\
& & BTS & 0.001 & 0.031 & 0.032 & 0.003 & 0.049 & 0.052 & 0.002 & 0.058 & 0.060 & 0.002 & 0.092 & 0.093 \\
& & PRC & 0.000 & 0.067 & 0.067 & 0.000 & 0.080 & 0.080 & 0.000 & 0.083 & 0.083 & 0.000 & 0.108 & 0.108 \\
& & BC & 0.015 & 0.039 & 0.054 & 0.032 & 0.034 & 0.066 & 0.040 & 0.023 & 0.062 & 0.059 & 0.015 & 0.074 \\
& & BCA & 0.009 & 0.046 & 0.055 & 0.029 & 0.040 & 0.068 & 0.035 & 0.025 & 0.060 & 0.055 & 0.016 & 0.071 \\
& & JAC & 0.003 & 0.049 & 0.052 & 0.010 & 0.047 & 0.057 & 0.010 & 0.038 & 0.048 & 0.016 & 0.033 & 0.049 \\
& & BSD & 0.002 & 0.046 & 0.048 & 0.009 & 0.046 & 0.055 & 0.009 & 0.039 & 0.048 & 0.016 & 0.033 & 0.049 \\
\hline \multirow{3}{*}{0.8} & CM & 0.004 & 0.057 & 0.061 & 0.009 & 0.046 & 0.055 & 0.014 & 0.040 & 0.054 & 0.018 & 0.031 & 0.049 \\
& & BTS & 0.001 & 0.033 & 0.034 & 0.004 & 0.027 & 0.031 & 0.005 & 0.039 & 0.043 & 0.005 & 0.033 & 0.038 \\
& & PRC & 0.000 & 0.043 & 0.043 & 0.001 & 0.042 & 0.042 & 0.001 & 0.043 & 0.044 & 0.003 & 0.039 & 0.041 \\
& & BC & 0.011 & 0.071 & 0.081 & 0.017 & 0.059 & 0.075 & 0.034 & 0.042 & 0.076 & 0.035 & 0.026 & 0.061 \\
& & BCA & 0.006 & 0.070 & 0.076 & 0.016 & 0.059 & 0.074 & 0.030 & 0.043 & 0.072 & 0.033 & 0.026 & 0.059 \\
& & JAC & 0.004 & 0.062 & 0.066 & 0.010 & 0.050 & 0.060 & 0.015 & 0.042 & 0.057 & 0.019 & 0.032 & 0.050 \\
& & BSD & 0.004 & 0.061 & 0.065 & 0.008 & 0.048 & 0.056 & 0.015 & 0.042 & 0.057 & 0.017 & 0.032 & 0.048 \\
\hline
\end{tabular}

